\title{
The Magnetic Properties of Electrical Steel for Rotating Machine according to the Specimen
}

\author{
Yun-Yong Choi, Jun-Woo Chin, and Jung-Pyo Hong* \\ Department of Automotive Engineering, Hanyang University, Seoul 04763, Korea
}

(Received 22 February 2016, Received in final form 30 March 2016, Accepted 31 March 2016)

\begin{abstract}
This paper analyzes the magnetic property according to the machined shape of steel material with non-oriented silicon steel (50PN470/50A470), that is most commonly used in the design of electrical equipment. Toward this end, specimens were produced and divided into Bar-Specimen (Epstein Frame Tester) and Ring-Specimen (Toroidal Ring Tester). The characteristics of the electrical Silicon steel were measured using the instruments solely dedicated to measuring each specimen. The core loss of the Bar-Specimen, which is commonly used, was found to be less than that of the Ring-Specimen. This is a very important design factor in achieving the objectives of improving the product efficiency and predicting the performance of electrical equipment. It serves as a critical point of view in order to reduce the error between design value and product value. A comparative analysis was conducted regarding various characteristics (Hysteresis, B-H characteristic, Iron loss, Minor loop, Coercive force, Residual magnetic flux density, etc.) of the electrical silicon steel considered in the design of the electrical equipment according to the specimen.
\end{abstract}

Keywords : electrical steel, Bar-Specimen, Ring-Specimen, magnetic properties

\section{Introduction}

At the present time when greater demand for eco-friendly products is seen worldwide, the efficiency improvement of the electrical equipment which accounts for more than half of the energy market emerged as an important issue. In particular, the efficiency improvement in terms of production and consumption of electrical generators and motors is an important key towards meeting the future energy demand. Globally, studies on the efficiency improvement of electrical equipment that consider the material properties based on the shape design will have been conducted by $2035[1,2]$.

Of these, almost all electric devices, like generators, motors and transformers exhibit different properties depending on the characteristics of the electrical silicon steel. In this light, a technique that can accurately measure the properties of the electrical silicon steel can be said to the core technology not only for producing the high quality electrical silicon steel but also for developing superior electrical equipment with high efficiency and high perfor-

(C)The Korean Magnetics Society. All rights reserved.

*Corresponding author: Tel: +82-2-2220-4466

Fax: +82-2-2220-4465, e-mail: hongjp@hanyang.ac.kr mance through the application of the measurement results to the performance prediction design of the electrical equipment [3]. In addition, a key point is how much energy loss the actual products can be reduced through the efficiency improvement of the electrical equipment, which is the ultimate goal of the performance prediction design [4].

Especially in the design of the electrical equipment (AC motor or actuators, etc.), the core loss of the steel used is the critical design aspect. The measurement only in consideration of the shape of the actual products, such as the modeling, analysis and measurement, is a very important factor $[5,6]$.

In general, the electrical silicon steel uses a standardized test method (e.g. Epstein Frame Measurement) to characterize its products from the viewpoint of core loss and magnetic property at a certain operating point. In this standardized test, a fixed laminated-type and a specimen (Bar-Specimen) are used, and a general machine shape is not taken into account. This method is one of the reasons for the inaccuracy of core losses in electrical machines. Yet another reason is the deterioration of the magnetic material in the general machine manufacturing process, that is an induced stress that occurs during the process of cutting and punching as well as loading and welding the 
laminated sheet, which ultimately affects the initial magnetization curve and hysteresis loop of the material, resulting in a number of errors in predicting changes in core loss of the steel $[7,8]$.

In this paper, a comparative analysis of $\mathrm{B}-\mathrm{H}$ characteristics and core loss measurements was performed using the dedicated measurement equipment (Epstein Frame Measurement vs. Toroidal Ring Tester) in relation to differences according to material processing type (BarSpecimen vs. Ring-Specimen) based on the $50 \mathrm{~Hz}$ sinusoidal exciting current and DC BIAS conditions with respect to the most commonly used 50PN470 (50A470) material [9-11].

\section{Measurement Methods}

\subsection{Theoretical background}

With regard to the magnetic hysteresis loop of the electrical silicon steel, if the magnetic intensity $(H)$ is increased in a situation where the specimen is not magnetized at all, the magnetic domain begins to arranged and magnetized to $a$ point of Fig. 1. If the strength of the magnetic field is gradually reduced to 0 point from the magnetized status, the magnetic flux density is decreased along the $a-b$ curve, rather than along the initial magnetization curve, and a part of the magnetic domain with the previous direction still remains as in ( $b$ point). A negative magnetic field ( $c$ point, $-H_{c}$ ) is needed for the magnetic field $(B)$ of the specimen to become 0 , and the negative magnetic field $(-H)$ arranges the magnetic domain in the opposite direction and the saturation occurs ( $d$ point). On the same principle, a symmetrical magnetic hysteresis loop is created in $e$ curve [12-14].

The magnetic flux density can be traditionally measured using B-Coil. If the current is applied to B-Coil, the magnetization occurs inside, when the following voltage is induced at both ends of B-Coil due to changes in the amount of magnetic flux that links B-coil.

$$
e=\oint_{\text {coil }} \vec{E} \cdot d \vec{l}=\frac{d \lambda}{d t}=N A \frac{d B}{d t}[\mathrm{~V}]
$$

$\vec{E}, \lambda$ represent the intensity of the induced electric field and the entire linkage magnetic flux. $N$ and $A$ represent the number of turns and cross-sectional area of B-coil, respectively. At this point, the magnetic flux density of the inside the B-coil is calculated as follows [15].

$$
B(t)=\frac{1}{N A} \int_{0}^{t} e(\tau) d \tau[\mathrm{T}]
$$

The strength of the magnetic field was measured using the exciting current method. The relationship between the magnetic field strength and exciting current in the magnetic closed circuit is represented using the Ampere's circuit law as follows.

$$
\oint_{c} \vec{H} \cdot d \vec{l}=N i[\mathrm{~A}]
$$

If the preceding formula is applied to Ring-Specimen, the relationship between the magnetic field strength and the applied current can be obtained as follows.

$$
H(t)=\frac{N}{l} i(t)=\frac{N}{2 \pi r} i(t)[\mathrm{A} / \mathrm{m}]
$$

Where $l$ represents the length of the effective magnetic flux, and $r$ the effective radius of Ring-Specimen as in

\begin{tabular}{|c|c|c|c|c|c|c|c|c|}
\hline \multirow{2}{*}{$\begin{array}{c}\text { Thickness } \\
\text { (mm) }\end{array}$} & \multirow{2}{*}{$\begin{array}{l}\text { Density } \\
\left(\mathrm{g} / \mathrm{cm}^{3}\right)\end{array}$} & \multicolumn{4}{|c|}{ Core loss $(\mathrm{W} / \mathrm{Kg})$} & \multicolumn{2}{|c|}{$\begin{array}{l}\text { Magnetic Flux } \\
\text { Density (T) }\end{array}$} & \multirow{2}{*}{$\begin{array}{c}\text { Fill Factor } \\
(\%)\end{array}$} \\
\hline & & $\mathrm{W} 10 / 50$ & $\mathrm{~W} 15 / 50$ & W10/60 & $\mathrm{W} 15 / 60$ & $\mathrm{~B} 25$ & B50 & \\
\hline 0.5 & 7.70 & 1.64 & $\begin{array}{c}3.55 \\
(\max 4.70)\end{array}$ & 2.06 & 4.56 & 1.61 & $\begin{array}{c}1.70 \\
(\min 1.64)\end{array}$ & 96.0 \\
\hline
\end{tabular}

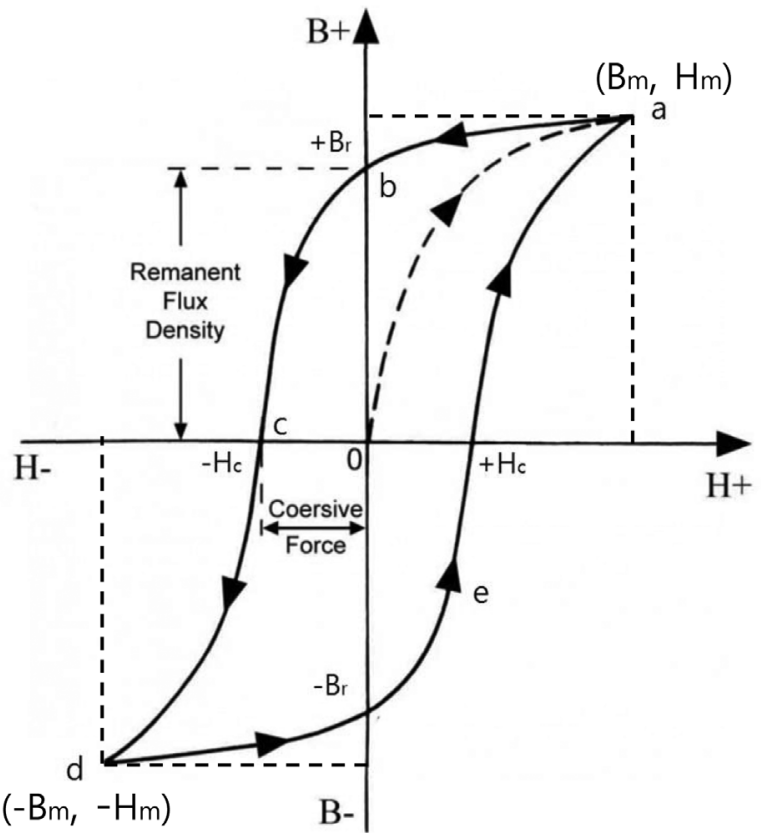

Fig. 1. Magnetic Hysteresis Loop.

Table 1. The material and magnetic properties based on S18 (50PN470, 50A470) specimen. 


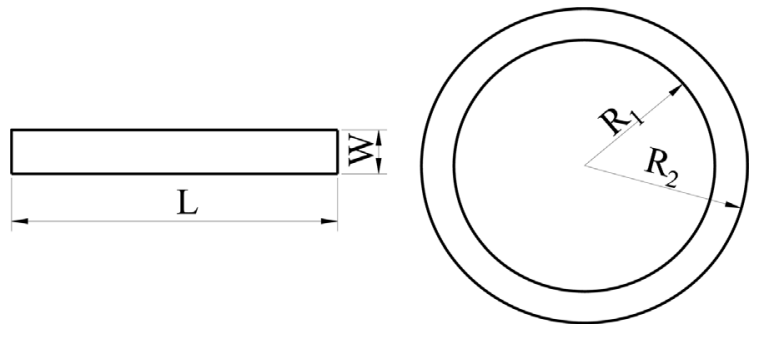

Fig. 2. The bar specimen and the ring specimen.

$\left(R_{1}+R_{2}\right) / 2$ of Fig. 2 . In the case of the Epstein Frame Measurement, $l$ is a structure in which the length of the effective magnetic flux is fixed to the IEC standard (94 $\mathrm{cm})$ [9].

The core loss occurring in the electrical silicon steel is caused by a temporal change of the magnetic intensity $(H)$ and magnetic flux density $(B)$ inside the electrical silicon steel, and if the temporal change of $B$ and $H$ is given, the calculation is made from Poynting's theorem as follows [16].

$$
P=\frac{1}{\rho T} \int \vec{H}(t) \frac{d \vec{B}(t)}{d t} d t[\mathrm{~W} / \mathrm{kg}]
$$

Where $P$ is [W/kg, specific iron loss], $B$ [T, magnetic flux density], $H\left[\mathrm{~A} / \mathrm{m}\right.$, magnetic intensity], $\rho\left[\mathrm{kg} / \mathrm{m}^{3}\right.$, material density], and $T$ [s, time period of $B$ and $H$ waveform].

\subsection{Measurement System}

For the magnetic hysteresis test of the electrical silicon steel, the Ring-Specimen for Toroidal Ring Tester and the Bar-Specimen for Epstein Frame Measurement were produced in accordance with the IEC test standards with steel materials based on Table 1 .

As shown in Fig. 2, the Bar-Specimen is a standard specimen with a thickness $(t)$ of $0.5 \mathrm{~mm}$, a width $(W)$ of $30 \mathrm{~mm}$, and a length $(L)$ of $290 \mathrm{~mm}$, and the RingSpecimen measures $0.5 \mathrm{~mm}$ in thickness $(t), 50 \mathrm{~mm}$ in inner radius $\left(R_{1}\right)$, and $65 \mathrm{~mm}$ in outer radius $\left(R_{2}\right)$ and was produced in $R_{2} \leq 1.4 R_{1}$ condition to ensure the uniformity of the distribution of the magnetic flux that occurs in the outer diameter.

In order to connect the produced specimens to each measuring equipment as shown in Fig. 3, the Bar-Specimen was inserted in a way of laminating each four Barspecimens to the center for each of the four coils of the Epstein Frame Measurement, and a cooling system was installed to minimize the error caused by the heat generation of each winding and maintain the insulation of the exciting winding. In order to connect the Ring-Specimen to the Toroidal Ring Tester, the primary exciting winding was wound by a single 300 -turn winding of wire with a

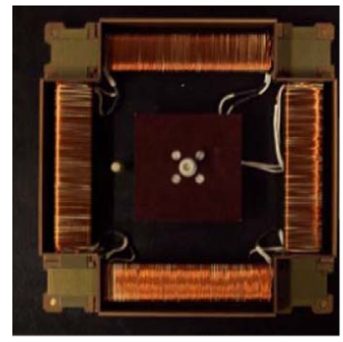

(a) Epstein Frame specimen

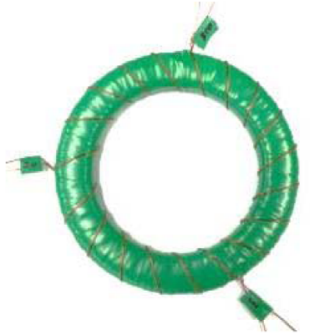

(b) Toroidal Ring specimen
Fig. 3. (Color online) Photograph of the Epstein Frame and the Toroidal Ring specimen.

diameter of $0.8 \mathrm{~mm}$, the secondary measuring winding by a single 20-turn winding of wire with a diameter of 0.8 $\mathrm{mm}$, and the third DC bias winding by a single 200-turn winding of wire with a diameter of $0.8 \mathrm{~mm}$ in a way of winding induction coils on the circumstances of the specimens in a state where 10 basic specimens are laminated. In addition, insulation tapes were wound around each winding for insulation and error against the generation of heat.

The final specimens made in Fig. 3 are connected to each terminal of the measuring devices as in Fig. 4. Figure 4 shows a brief circuit diagram of each measuring device, such as Toroidal Ring Tester (B-H Analyzer SY8258, manufactured by IWATSU Test instruments corp.) and Epstein Frame Tester 700/700/80 [Turns] coil specifications produced in accordance with the IEC standard.

For measurements, $B_{\max }$ is set in consideration of the limitations of the equipment and the saturation state of the
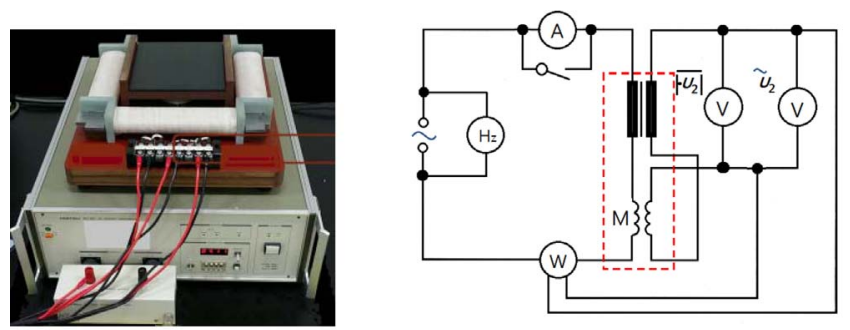

a) The Epstein Frame tester and circuit
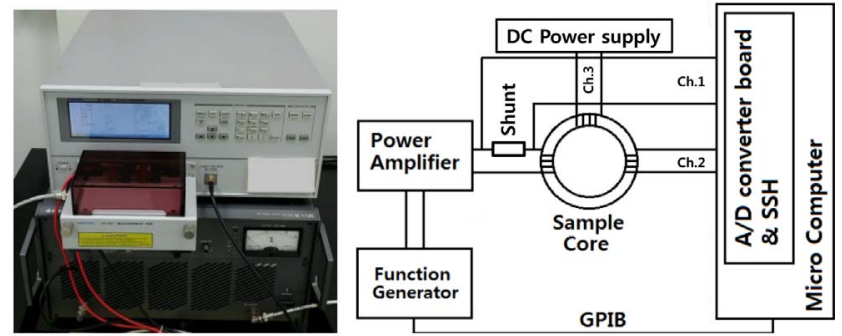

b) Toroidal Ring tester and circuit

Fig. 4. (Color online) Picture and circuit of a) the Epstein Frame tester and b) the Toroidal Ring tester. 
hysteresis loop after measuring the hysteresis loop. In this experiment, the hysteresis loop was measured by changing $\mathrm{H}$ values after setting $B_{\max }$ to $1.4 \mathrm{~T}$ and the setting unit of $B_{e x}$ (magnetic flux density measurement value) to $0.1 \mathrm{~T}$. The magnetic hysteresis loop was measured by repeating $B_{e x}$ from $0.1 \mathrm{~T}, 0.2 \mathrm{~T}, \ldots$. , up to $1.4 \mathrm{~T}$. Based on the measurement results, B-H curve characteristics and core losses are calculated [17-19].

\section{Measurement Result}

Magnetic properties were measured in a sinusoidal magnetic field condition (Sinusoidal $H$ condition), and the characteristics of the steel were compared based on the measurement results. In the experiment, the frequency of the exciting current was maintained at $50 \mathrm{~Hz}$ [20].

Figures 5 and 6 show the measured hysteresis loops of Bar-Specimen and Ring-Specimen. In these two figures, $B_{e x}$ ranges from 0.1 to $1.4 \mathrm{~T}$. A comparison of B-H curve of the two specimens based on the same $B_{e x}$, value revealed that $H_{m}$ value of Ring-Specimen is larger than that

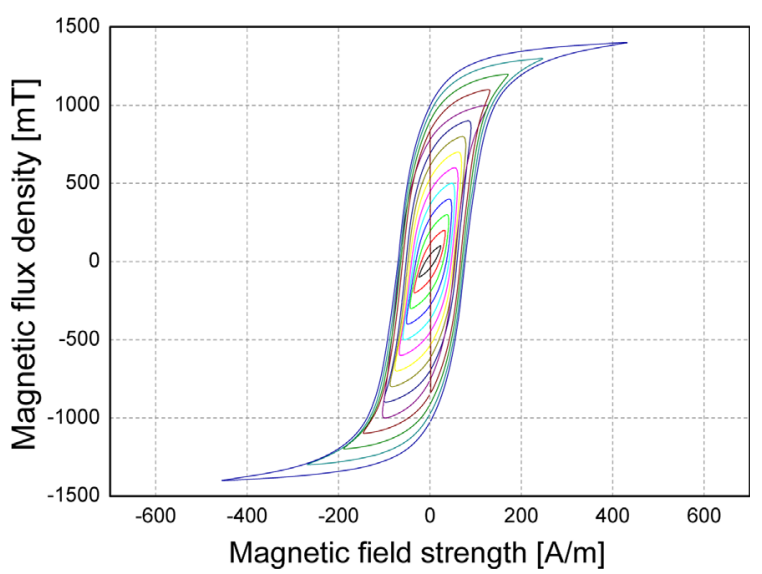

Fig. 5. (Color online) Hysteresis loops of the bar specimen.

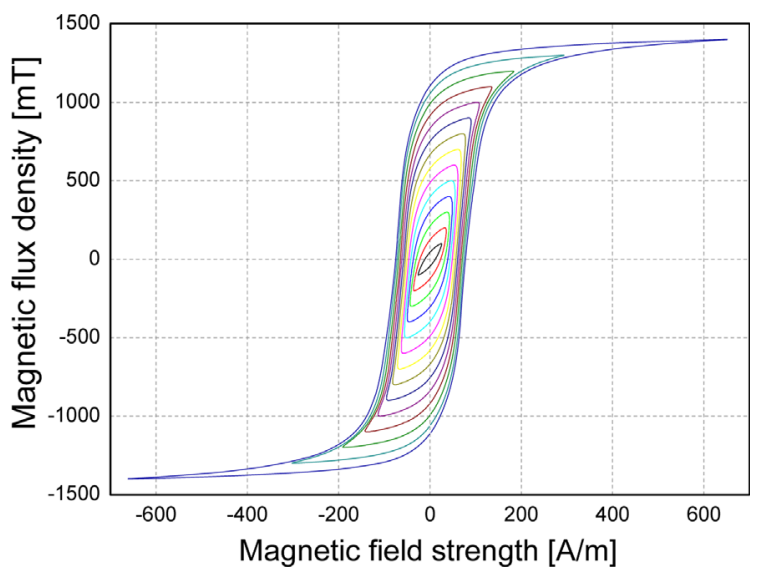

Fig. 6. (Color online) Hysteresis loops of the ring specimen.

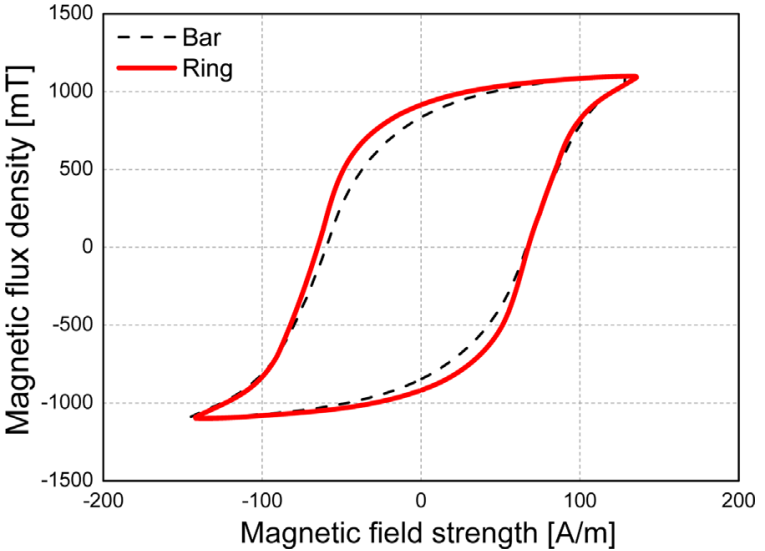

Fig. 7. (Color online) Hysteresis loop of the bar specimen and the ring specimen $\left(B_{e x}=1.1 \mathrm{~T}\right)$.

of Bar-Specimen. And the slop of the B-H curve is slightly smaller in Bar-Specimen than in Ring-Specimen. Figure 7 shows the details of changes in hysteresis loop based on the results of comparing the hysteresis loop of Bar-Specimen and Ring-Specimen when $B_{e x}$ is $1.1 \mathrm{~T}$. The solid line shows the hysteresis loop of Ring-Specimen, and short dotted line is the hysteresis loop of Bar-Specimen. The coercivity of hysteresis loop $\left(H_{c}\right)$ and residual induction $\left(B_{r}\right)$ appear slightly larger in Ring-Specimen than in Bar-Specimen.

From Fig. 7, it can be seen that the magnetic properties of Ring-Specimen were clearly worse than those of BarSpecimen. Therefore, the core loss also shows a tendency to be greater. This seems to be due to the difference in the stress state according to the processing of materials [21, 22]. Figure 8 shows the relationship of iron loss according to the magnetic flux density of Bar-Specimen and Ring-Specimen. While each core loss of the two specimens shows little difference, the core loss of Ring-Specimens

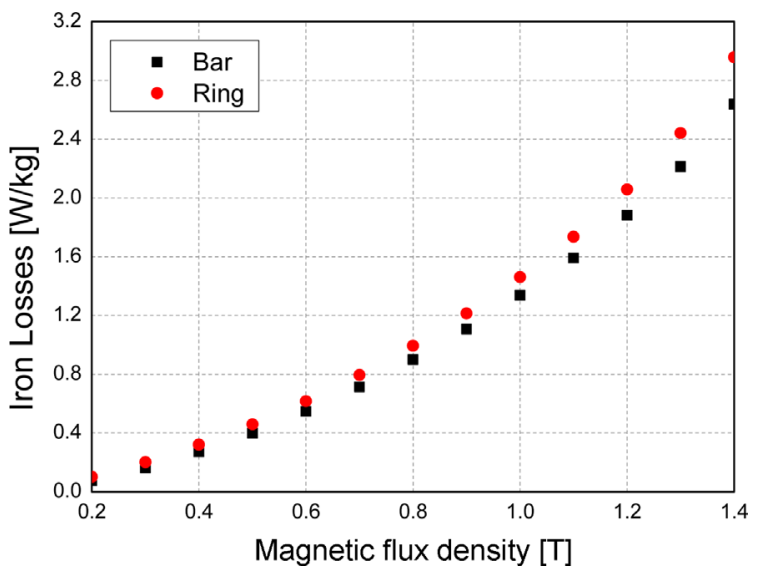

Fig. 8. (Color online) Iron loss $\left(P_{x}\right)$ of the bar specimen and the ring specimen. 


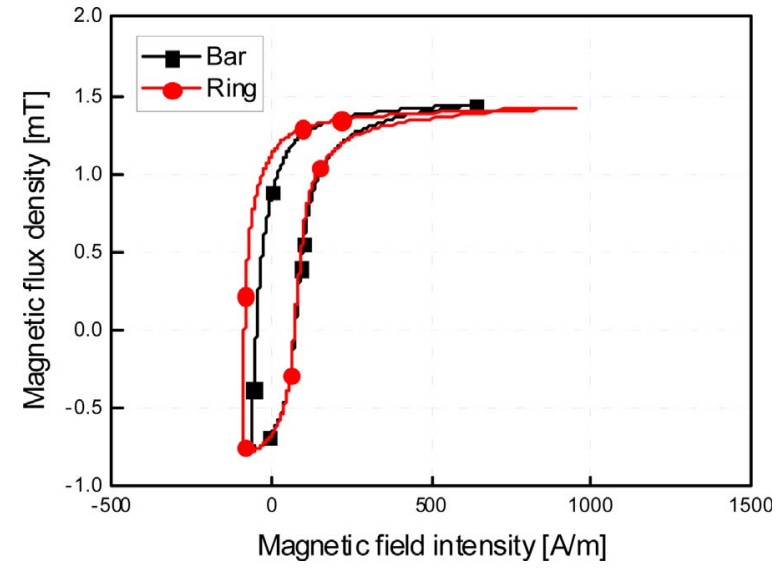

Fig. 9. (Color online) Minor loop of the bar specimen and the ring specimen.

shown to be larger. Figure 9 shows a minor loop in the case of a DC-bias of up to $100 \mathrm{~A} / \mathrm{m}$ in the magnetic flux density condition of Fig. 7. This is because in the case of Ring-Specimen, many harmonic components are contained in the magnetic flux density, and the eddy current loss they produce is added to the magnetic hysteresis loss.

Therefore, the eddy current loss caused by the magnetic flux density component of the harmonic component in the same magnetic field strength is the difference between the two kinds of core losses shown in Fig. 8, which is equal to the difference in the area of the magnetic hysteresis loop in Fig. 7. When $B_{e x}$ Is about 1.4T, Ring-Specimen was found to be about $12 \%$ higher.

Figure 10 shows the relationship between $B_{e x}$ and $H_{c}$ (coercive force) of Bar-Specimen and Ring-Specimen. Ring-Specimen showed higher $H_{c}$ with a constant difference according to the changes in $B_{e x}$ values overall.

As shown in Fig. 11, $B_{e x}$ and $B_{r}$ (residual induction) characteristics of Bar-Specimen and Ring-Specimen become

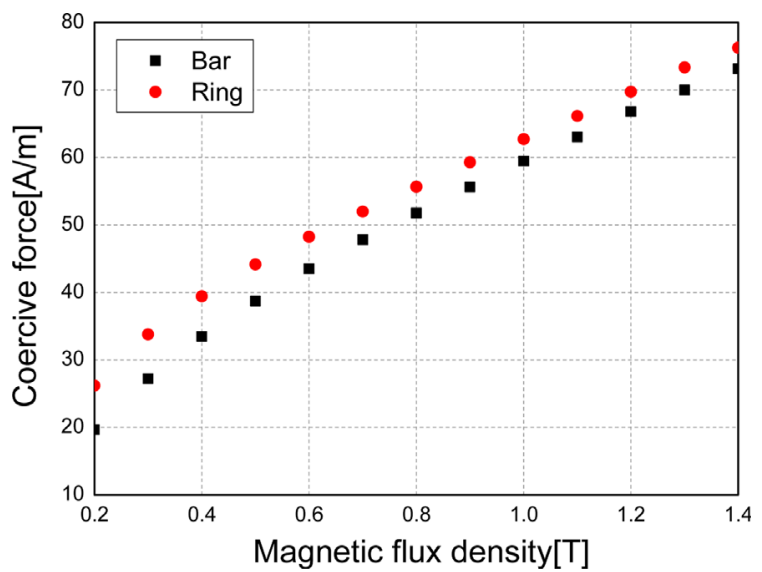

Fig. 10. (Color online) Coercive force $\left(H_{c}\right)$ of of the bar specimen and the ring specimen.

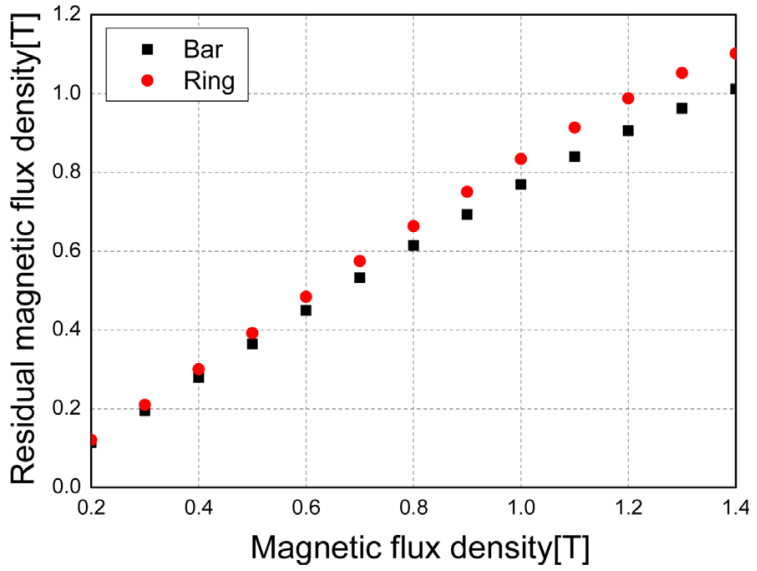

Fig. 11. (Color online) The residual magnetic flux density $\left(B_{r}\right)$ of the bar specimen and the ring specimen.

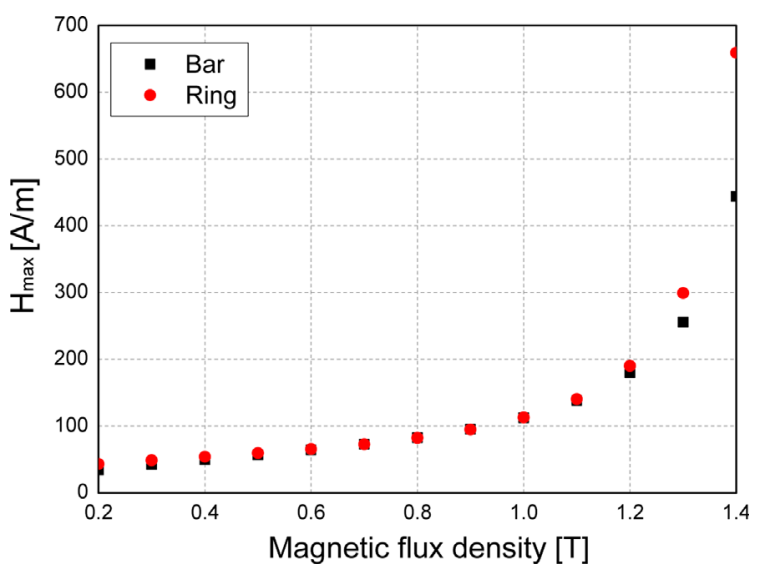

Fig. 12. (Color online) The maximum excitation magnetic field strenth $\left(H_{\max }\right)$ of the bar specimen and the ring specimen.

gradually higher with a constant difference according to the changes in $B_{e x}$ values, and a difference of up to $9 \%$ is shown when $B_{e x}$ is $1.4 \mathrm{~T}$.

Figure 12 shows the relationship of $H_{m}$ between BarSpecimen and Ring-Specimen due to the increase of $B_{e x}$ The two specimens show almost similar slope until $B_{e x}$ becomes 1.1T, but Ring-Specimen shows a steeper slope based on the $B_{e x} 1.2 \mathrm{~T}$. When $B_{e x}$ is $1.4 \mathrm{~T}$, which is the maximum value of this experiment, the change of $H_{m}$ is greatest, and Ring-Specimen shows about $215 \mathrm{~A} / \mathrm{m}$ (49 $\%)$ larger value than Bar-Specimen

Table 2 shows the magnetic properties of Ring-Specimen proportionally based on the magnetic properties of

Table 2. The difference of the measured magnetic properties of the Ring-Specimen compared with Bar-Specimen $\left(B_{e x}=1.4 \mathrm{~T}\right)$.

\begin{tabular}{lcccc}
\hline \hline & $P \mathrm{x}$ & $H \mathrm{c}$ & $B \mathrm{r}$ & $H \mathrm{~m}$ \\
\hline Ratio [\%] & 12.1 & 4.3 & 8.9 & 48.6 \\
\hline
\end{tabular}


Bar-Specimen. As shown in this table, the measured magnetic properties of Ring-Specimen seem worse than those of Bar-Specimen. This is because the use of a cylindrical specimen close to the shape of the actual product is thought to have a greater effect on the eddy current loss, rather than the use of a standard specimen.

\section{Conclusion}

In this paper, specimens were produced using the electrical silicon steel, which is commonly used in the design of the electrical equipment and divided into a standard specimen (Bar-Specimen) and a specimen with a similar shape to the actual product (Ring-Specimen) according to the machined shape of the steel. And then, the magnetic properties were measured, and a comparative analysis of the measurement results confirmed that the magnetic properties of Ring-Specimen appear worse than those of Bar-Specimen.

It is predicted that the cause of this phenomenon may include the impact of processing deterioration during the process of specimen production, the differences in characteristics of equipment itself and eddy current loss according to the shape (thickens or size) and the effects in measuring harmonic characteristics. However, the important conclusion of this paper is that in general, the standard bulk-type specimen on sale and the specimen with a similar shape to the actual product show differences in the magnetic properties, and the measurement of specimen suitable for the shape of the actual product of the electrical equipment is needed for more accurate efficiency and performance predictions of the electrical equipment.

In addition, there is a need to establish the standardization of the specimen production procedures and measurement techniques in order to obtain material characteristics close to those of the actual product.

The findings of this study are expected to be utilized in the design of the electrical silicon steel for the actual product. For this, the measurement and analysis on the changes in the magnetic properties of more various steel types (thickness, size) and specimen shapes should precede the other elements, and finally the effects on the performance of the electrical equipment should also be investigated.

\section{Acknowledgment}

This research was supported by the MSIP (Ministry of
Science, ICT and Future Planning), Korea, under the CITRC (Convergence Information Technology Research Center) (IITP-2015-H8601-15-1005) supervised by the IITP (Institute for Information \& communication Technology Promotion).

\section{References}

[1] International Energy Agency, World energy outlook, Organization for Economic Co-operation and Development, Paris, France (2013) pp. 623-625.

[2] B. Mecrow and A. Jack, Energy Policy (2008) pp. 43364341.

[3] Y. Kai, S. Zeze, T. Todaka, and M. Enokizono, IEEE Trans. Magn. 49, 5 (2013).

[4] Y. Zhang, Y. Liu, Y. Li, D. Xie, and B. Bai, IEEE Trans. Magn. 50, 2 (2014).

[5] A. Krings, M. Cossale, A. Boglietti, A. Cavagnino, and J. Soulard, IEEE Energy Conversion Congress and Exposition (ECCE), Pittsburgh, USA (2014).

[6] A. Krings, S. Nategh, A. Stening, H. Grop, O. Wallmark, and J. Soulard, International Conference Magnetism and Metallurgy (WMM), Gent, Belgium (2012).

[7] A. Krings, Iron Losses in Electrical Machines, Doctoral Thesis, Stockholm, Sweden (2014).

[8] Hwi Jun Kim, J. Kor. Magn. Soc. 21, 2 (2011).

[9] IEC Standard Publication 60404-2 (2008).

[10] J. Sievert, J. Magn. Magn. Mater. 215 (2000).

[11] M. Enokizono, Y. Takeshima, and H. Matsuo, J. Magn. Soc. Jpn. 24, 4 (2000).

[12] Seung-Hee Chai, Ji-Hyun Kim, Sung-Il Kim, and J. P. Hong, J. Kor. Magn. Soc. 20, 3 (2015).

[13] B. Z. Chen, S. Yan, Y. Z. Ju, X. C. Zhang, M. Yue, J. Xia, and G. P. Zhao, J. Kor. Magn. Soc. 20, 1 (2015).

[14] Hyun-Seok Whang, Sang-Jun Yun, Joon Moon, and SugBong Choe, J. Kor. Magn. Soc. 20, 1 (2015).

[15] Gordon R. Slemon, Electrical machine and drives. Addison-Wesley publishing company Inc. (1992) pp. 2-31.

[16] K. Atllah and D. Howe, IEEE Trans. Magn. 29, 6 (1993).

[17] Shiuk Chung, Daehyun Koo, Choongkyu Han, and Jiyoung Lee, J. Kor. Magn. Soc. 19, 2 (2014).

[18] Seul-Ki Nam, Sun-Gyu Moon, Keun Yong Sohn, and Won-Wook Park, J. Kor. Magn. Soc. 19, 4 (2014).

[19] Sung-Ho Lee, Yong-Jae Kim, Kyu-Seok Lee, and SungJin Kim, J. Kor. Magn. Soc. 20, 4 (2015).

[20] S. Y. Park, K. S. Ryu, J. K. Yi, and J. S. Park, J. Kor. Magn. Soc. 16, 4 (2006).

[21] M. S. Lim, J. H. Kim, and J. P. Hong, IEEE Trans. Magn. 51, 11 (2015).

[22] Y. Kai, Y. Tsuchida, T. Todaka, and M. Enokizono, IEEE Trans. Magn. 48, 4 (2012). 